\title{
Measuring Distributional Inequality: Relative Body Mass Index Distributions by Gender, Race/Ethnicity, and Education, United States (1999-2006)
}

\author{
Brian C. Houle \\ Department of Sociology, University of Washington, 211 Savery Hall, Box 353340, Seattle, WA 98195-3340, USA \\ Correspondence should be addressed to Brian C. Houle, bhoule@uw.edu
}

Received 5 September 2010; Revised 17 December 2010; Accepted 21 December 2010

Academic Editor: Aron Weller

Copyright () 2010 Brian C. Houle. This is an open access article distributed under the Creative Commons Attribution License, which permits unrestricted use, distribution, and reproduction in any medium, provided the original work is properly cited.

\begin{abstract}
Few studies consider obesity inequalities as a distributional property. This study uses relative distribution methods to explore inequalities in body mass index (BMI; kg/m²). Data from 1999-2006 from the National Health and Nutrition Examination Survey were used to compare BMI distributions by gender, Black/White race, and education subgroups in the United States. For men, comparisons between Whites and Blacks show a polarized relative distribution, with more Black men at increased risk of over or underweight. Comparisons by education (overall and within race/ethnic groups) effects also show a polarized relative distribution, with more cases of the least educated men at the upper and lower tails of the BMI distribution. For women, Blacks have a greater probability of high BMI values largely due to a right-shifted BMI distribution relative to White women. Women with less education also have a BMI distribution shifted to the right compared to the most educated women.
\end{abstract}

\section{Introduction}

More than one-third of USA adults were obese in 2007-2008, including $32.2 \%$ of men and $35.5 \%$ of women [1]. Overall the BMI distribution has shifted to the right, coupled with a greater increase in the prevalence of those who are extremely obese [2]. The increase in obesity due to this distributional shift translates to an increased chronic public health burden, as obesity is a risk factor for Type 2 diabetes, coronary heart disease, and several other diseases and conditions [3].

Obesity tends to increase with age for adults [2]. Obesity varies by race/ethnic group among women-both nonHispanic Blacks and Mexican-Americans have a higher prevalence of obesity [4]. Among adult women, poverty, and low educational levels are also associated with a higher prevalence of obesity $[5,6]$. However, little systematic variation by socioeconomic status (SES) has been observed among men. Until recently there was also little systematic variation by race/ethnicity observed among men $[1,2]$. Among young Black men, there is a positive association between education and obesity [7]. In a review of SES and obesity, Sobal and Stunkard found in the USA 12 studies showing a positive association between SES and obesity, 12 studies showing an inverse association, and 3 studies with no association among men [8]. Another study used the concentration index to assess obesity inequalities by income; they found a positive association among Black men and an inverse association among White men [9]. A recent review noted the complex relationships between gender, race/ethnicity, and SES particularly for men in the USA [10]. The mechanisms explaining the relative lack or mixed findings of social and race/ethnic inequalities among men compared to those observed among women are unclear; studies suggest mechanisms predicting similar and dissimilar inequalities by gender.

In order to adjudicate between these competing explanatory mechanisms, we need to be able to summarize inequality in obesity both within and between groups. Current obesity research summarizes these inequalities as differences in means or proportions, while other approaches based on regression focus on modeling the conditional mean (e.g., [4]). Since obesity inequality is a property of the body mass index (BMI) distribution, a focus on distributional methods can yield insights into the patterns in the available data. 
Contoyannis and Wildman summarize two key reasons to explore BMI distributions instead of just an examination of obesity: first, the BMI distribution recognizes that changes in risk are not typically discrete at obesity thresholds; second, while obesity rates are related to the BMI distribution, many different distributions can generate the same obesity rates [11]. Comparing differences across the entire distribution can also yield a detailed picture of distributional change, and can pose questions to guide further research. For instance, perhaps the mechanisms driving inequality in different parts of the distribution are different, or that between group gaps are driven by different factors at different points along the distribution. Current research is silent on BMI inequality within groups and limited on BMI inequality between them.

This study uses relative distribution techniques to analyze BMI inequalities both within and between different populations. Described in detail elsewhere [12], the relative distribution is effectively a transformation of two distributions into a single distribution that facilitates comparison. This study's goal is to analyze the relative distributions of BMI to assess gender-specific obesity inequalities over time between and within education and race/ethnic subgroups.

\section{Materials and Methods}

2.1. Participants and Measures. Non-Hispanic Whites and Blacks aged 20 years or older with valid height, weight, and education data were analyzed from the continuous (19992006) National Health and Nutrition Examination Survey [13]. NHANES is a complex, multistage probability sample of the civilian USA population. NHANES data are collected by interviews conducted in subjects' homes and standardized physical examinations conducted in mobile examination centers. Race/ethnicity and number of years of education are self-reported by the participants. Height and weight data are collected during the physical examination using standardized protocols and calibrated equipment. Body mass index was calculated as weight in kilograms divided by the square of height in meters. The use of measured BMI overcomes a weakness of relative distribution methods since they are not robust to data where respondents report round numbers rather than exact values (8). This effect can substantially alter the quantile aspects of a distribution.

2.2. Analysis. Relative distributions were created in $\mathrm{R}$ using the reldist package with the NHANES survey weights to account for differential nonresponse, noncoverage, and to adjust for over-sampling [14]. For a succinct summary of the method used in this paper see Contoyannis and Wildman [11]. The relative distribution compares two distributions as one moves along the BMI scale. For example, in the discrete case, we take the BMI distribution of the referent group and divide it into deciles. The relative distribution then measures the ratio of the fraction of the comparison group to the fraction of the referent group in each of these deciles. If $16 \%$ of the comparison group fell into the lowest BMI decile as defined by the referent group, then the relative distribution value would be $1.6(16 \% / 10 \%)$. If the comparison and referent groups had the same distribution, the relative distribution would take a value of 1.0 throughout (i.e., a uniform distribution).

Analyses were conducted separately for men and women due to gender interaction effects with the NHANES survey years [4]. For the adjudication of hypotheses underlying the comparison, some distributions were decomposed into location and shape differences. Location differences are presented as the relative distribution of the reference group locationadjusted to have the same median as the comparison group $\left(f_{0 \mathrm{~L}}\right)$ relative to the reference group $\left(f_{0}\right)$. This summarizes the extent to which the relative distribution is explained by a shift in the BMI distribution in the comparison compared to the referent group. Shape effects are presented as the relative distribution of the comparison group $(f)$ relative to the reference group location-adjusted to have the same median as the comparison group $\left(f_{0 \mathrm{~L}}\right)$. This summarizes the differences that remain after a location difference, consisting of spread, skew, and other distributional characteristics [12].

Some distributions were adjusted for changes in covariate composition, by creating an adjusted population that matches the covariate distribution of the referent group [12]. Besides graphical comparisons of differences between distributions, comparisons between distributions were also statistically summarized using the entropy statistic; this measures how different the distributions are from each other (where zero equals equivalence) [15]. Three entropy measures can be calculated: (1) overall entropy: the divergence between the comparison and reference groups; (2) divergence between the location-adjusted reference group and the reference group; (3) divergence between the comparison group and the location-adjusted reference group. These measures are all based on the Kullback-Leibler divergence measure:

$$
D\left(F ; F_{0}\right)=\int_{-\infty}^{\infty} \log \left(\frac{f(x)}{f_{0}(x)}\right) d F(x)=\int_{0}^{1} \log (g(r)) g(r) d r
$$

where $g(r)$ is the relative probability density function.

To summarize distributional polarization, the median relative polarization (MRP) index was used [15]. This measure isolates distributional inequalities not due to location shifts (it is location-adjusted). The MRP is scaled between - 1 to 1 , where zero represents no differences in distributional shape while positive values represent more polarization and negative values represent less polarization. In other words, positive values represent increases in the tails of the distribution, while negative values represent convergence to the center of the distribution.

$$
\operatorname{MRP}\left(F ; F_{0}\right)=4 \int_{0}^{1}\left|r-\frac{1}{2}\right| g_{0 L}(r) d r-1,
$$

where the MRP is the mean absolute deviation around the median of the location-matched relative distribution $\left(g_{0 \mathrm{~L}}\right)$ and weighted by $|r-1 / 2|$ (the distance from the center) to emphasize deviations in the tails [15].

The MRP is also decomposed into the upper and lower polarization index, representing the contributions from the upper and lower tails of the distribution. These 
TABLE 1: Un-weighted sample sizes by gender, education, and race-ethnic group, United States (1999-2006).

\begin{tabular}{lcccccccc}
\hline & \multicolumn{2}{c}{ Less than High School } & \multicolumn{2}{c}{ High School or GED } & \multicolumn{2}{c}{ Some college } & \multicolumn{2}{c}{ Bachelors or greater } \\
\hline & NH Whites & NH Blacks & NH Whites & NH Blacks & NH Whites & NH Blacks & NH Whites & NH Blacks \\
\hline Men & 778 & 617 & 1,200 & 422 & 1,233 & 475 & 1,214 & 228 \\
Women & 737 & 640 & 1,331 & 442 & 1,455 & 608 & 1,226 & 262 \\
\hline
\end{tabular}

Abbreviations: $\mathrm{NH}=$ Non-Hispanic. Data from the National Health and Nutrition Examination Survey.

decompositions are also scaled from -1 to 1 and have similar interpretations to the MRP

$$
\begin{aligned}
& \operatorname{URP}\left(F ; F_{0}\right)=8 \int_{1 / 2}^{1}\left|r-\frac{1}{2}\right| g_{0 L}(r) d r-1, \\
& \operatorname{LRP}\left(F ; F_{0}\right)=8 \int_{0}^{1 / 2}\left|r-\frac{1}{2}\right| g_{0 L}(r) d r-1 .
\end{aligned}
$$

\section{Results}

I first analyzed distributional change within groups to assess if covariates were giving different returns to parts of the distribution over time. For both men and women, there was little change within race/ethnic or education subgroups from 1999-2002 compared to 2003-2006 (results not shown). For non-Hispanic Black men and women, there was a slight increase in the proportion of cases with high BMI values. Given these results, I merged the two time periods to cover eight years to help reduce the effects of distributional instability due to small sample sizes in a decile. Table 1 summarizes the unweighted sample sizes by included covariates.

To help motivate the use of relative distributions, Figure 1 presents the probability density functions for non-Hispanic Black and White men. Some aspects of the relative BMI distribution are apparent: Black men have relatively greater cases in the upper and lower tails of the distribution; the modes of the distributions are also similar between the two groups. However, comparisons between the groups are not easily quantified. For instance, how much of the difference is captured by a location shift? Is there evidence of polarization and are the upper and lower tails of the distribution changing in similar ways? What is the role of education? We can use relative distribution methods to address these questions.

Figure 2 presents the gender-specific relative distributions comparing non-Hispanic Blacks to Whites and their decompositions into differences in median and shape. Adjusting for age did not substantively alter the results and are not presented. For men, panel (a) shows the relative distribution for the two distributions presented in Figure 1. Non-Hispanic Blacks have a more polarized relative distribution in both tails (see Table 2 for summary measures and measures of error). Roughly 1.5 times as many Blacks are in the bottom BMI decile defined by Whites. Further, about 30\% more Blacks than Whites were in the extreme obesity category. The median difference effect in panel (b) represents what the relative density would have looked like if there was no change in shape (note that due to rescaling the entropies will not sum to the overall value). The effects are

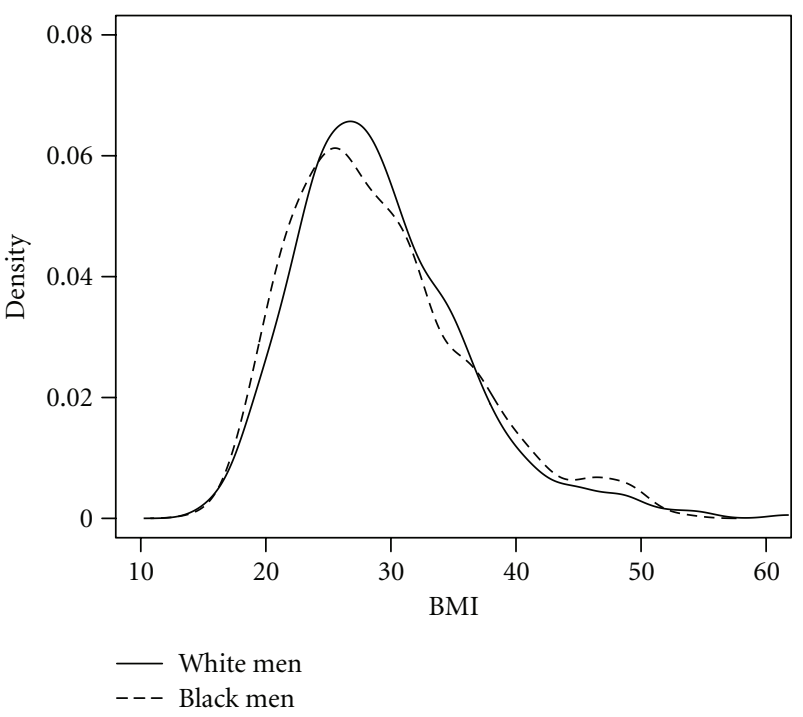

Figure 1: Probability density functions of body mass index for non-Hispanic Black and White men (1999-2006). Data from the National Health and Nutrition Examination Survey.

small, roughly explaining $16 \%$ of the difference between the BMI distributions. Adjusting for the median difference effect in panel (c) shows that the inequality observed in panel (a) is largely a product of shape differences (contributing roughly $74 \%$ of the difference between the distributions), with more Black men being at the extremes of the BMI distribution.

For women, Figure 2(d) (bottom) shows that nonHispanic Blacks have a greater proportion of the distribution in the obese BMI ranges compared to Whites (see Table 3 for summary measures and measures of error). The median effect decomposition in panel (e) is quite large, reproducing most of the observed relative distribution in the upper tail. Note, however, that the lower tail is not well reproduced by the median shift, being substantially lower than observed. The shape effects in panel (f) is concentrated in the lowest decile and shows a greater proportion of Blacks who are in the lowest decile operating alone (without the median effect) it would have increased the number of non-Hispanic Black women in the lowest BMI decile by about $80 \%$. From Figure 2, the relative distribution is polarized by race/ethnicity for men; for women, the BMI distribution is shifted to the right for non-Hispanic Blacks compared to non-Hispanic Whites.

Figure 3 presents the gender-specific relative distributions comparing those with a college education to other education subgroups, adjusted for age. For men, those with 


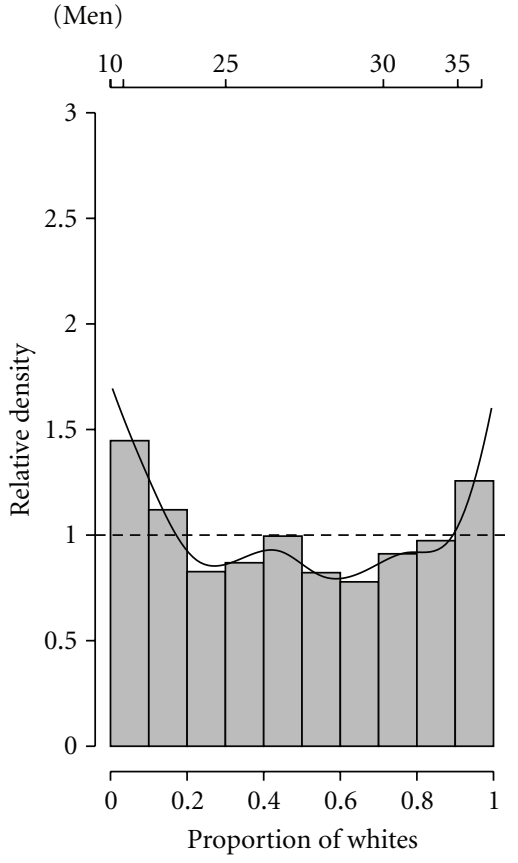

(a) Unadjusted entropy $=0.0228$

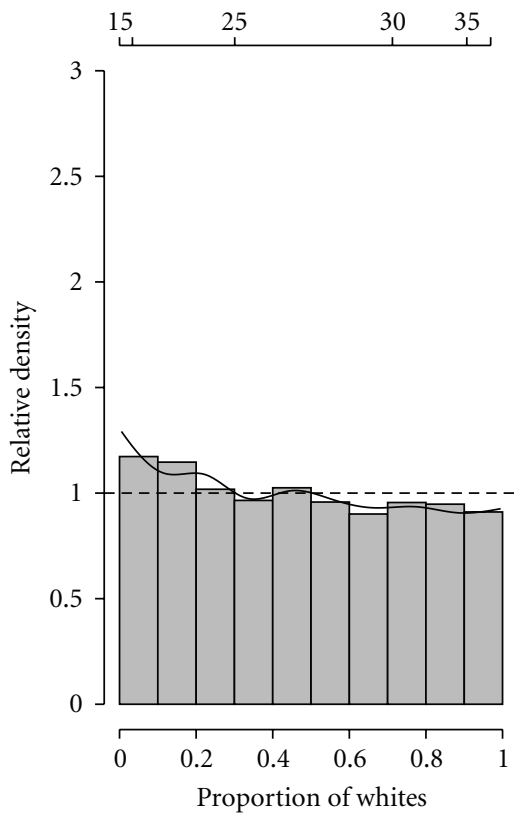

(b) Median difference effect entropy $=0.00378$

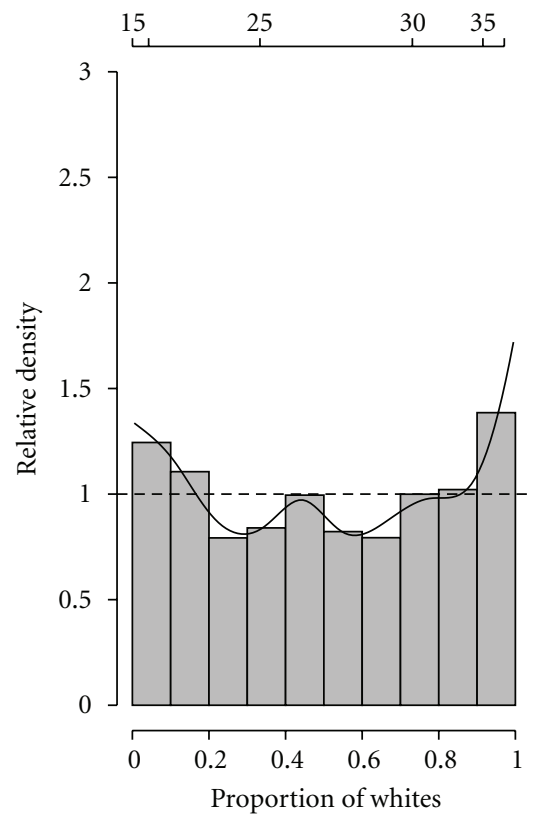

(c) Median-adjusted entropy $=0.0169$

(Women)

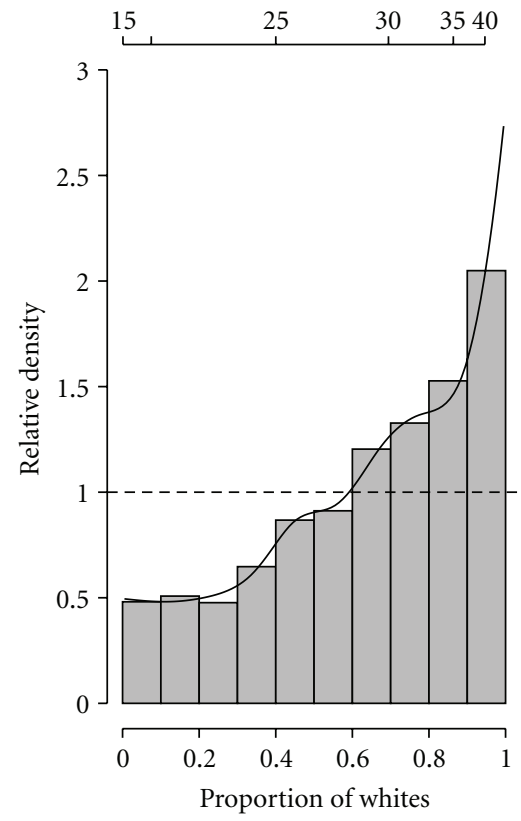

(d) Unadjusted entropy $=0.122$

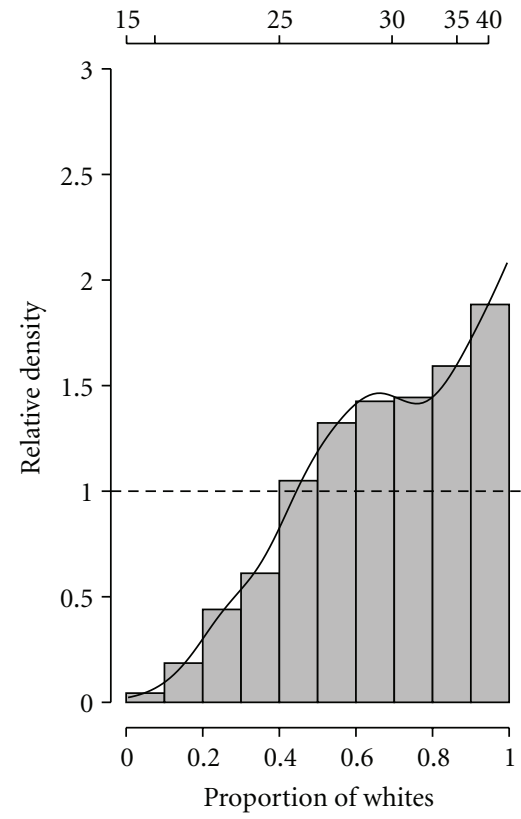

(e) Median difference effect entropy $=0.229$

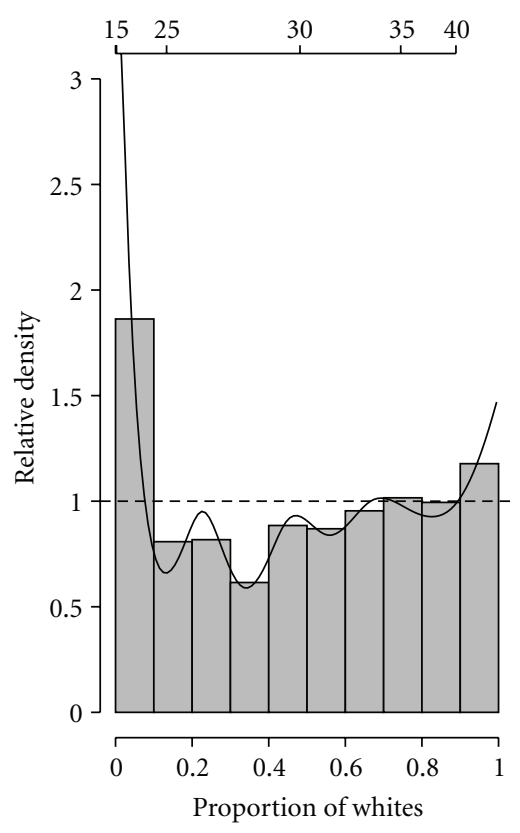

(f) Median-adjusted entropy $=0.0803$

FIGURE 2: Decomposing relative body mass index distributions (1999-2006) between non-Hispanic Black and White men (top) and women (bottom) into impact of changes in medians and changes in shape. (a,d) Unadjusted relative body mass index density; (b,e) effect of median difference in body mass index between race/ethnic subgroup; (c,f) median-adjusted relative body mass index density. Deciles chart superimposed on relative densities. Data from the National Health and Nutrition Examination Survey.

less than a high school diploma have a polarized distribution relative to the college educated (top panel (a); Table 2). Men with a high school diploma (top panel (b)) or some college (top panel (c)) have relatively more cases in the obese BMI values compared to the college educated. For instance, roughly 1.7 times as many men with a high school diploma or GED are in the top BMI decile of those with a college education.

For women, each lower education category has relatively more cases in the obese BMI values compared to the 


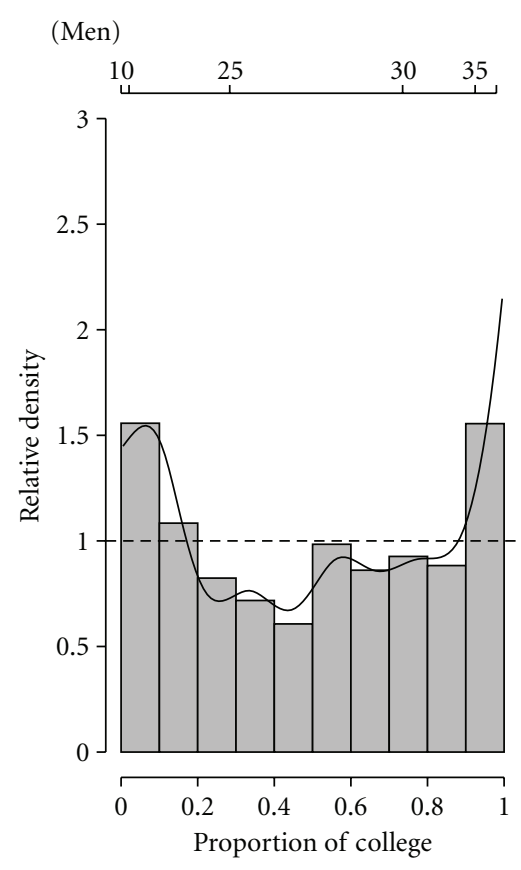

(a) Less HS-college entropy $=0.0456$

(Women)

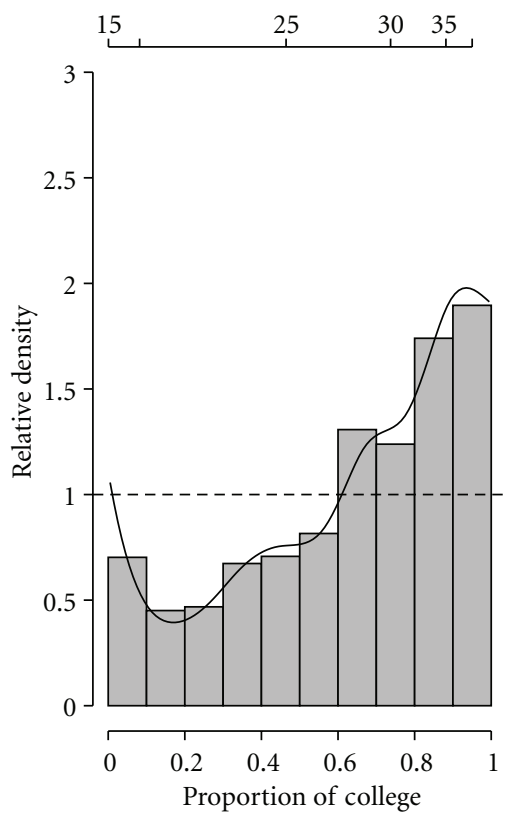

(d) Less HS-college entropy $=0.123$

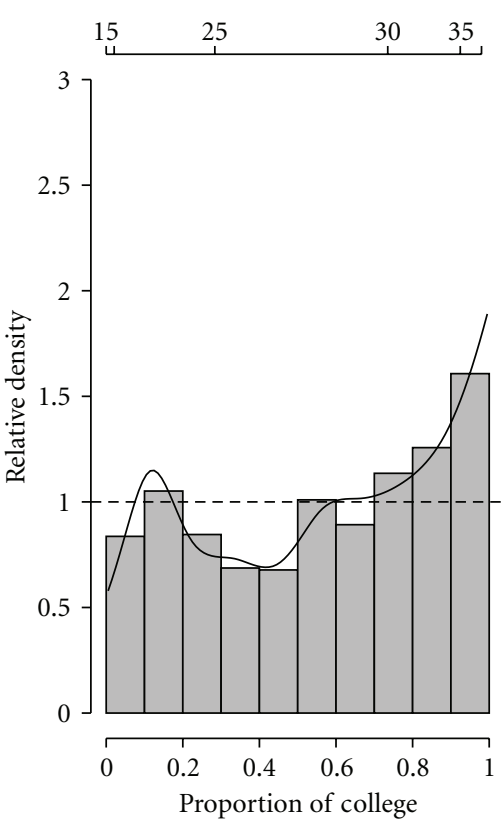

(b) HS-college entropy $=0.0354$

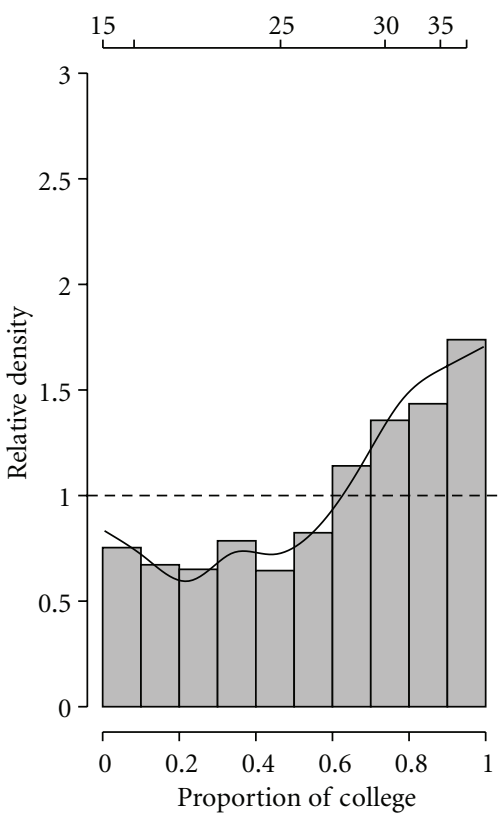

(e) HS-college entropy $=0.0664$

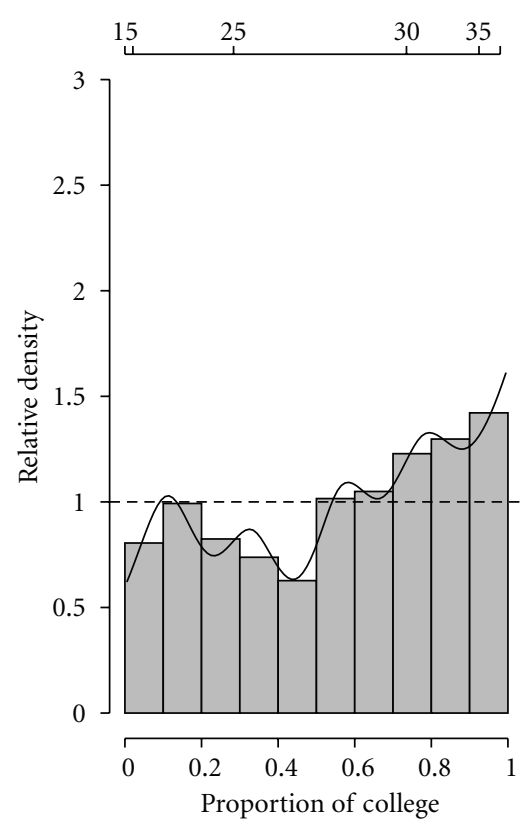

(c) Some college-college entropy $=0.0291$

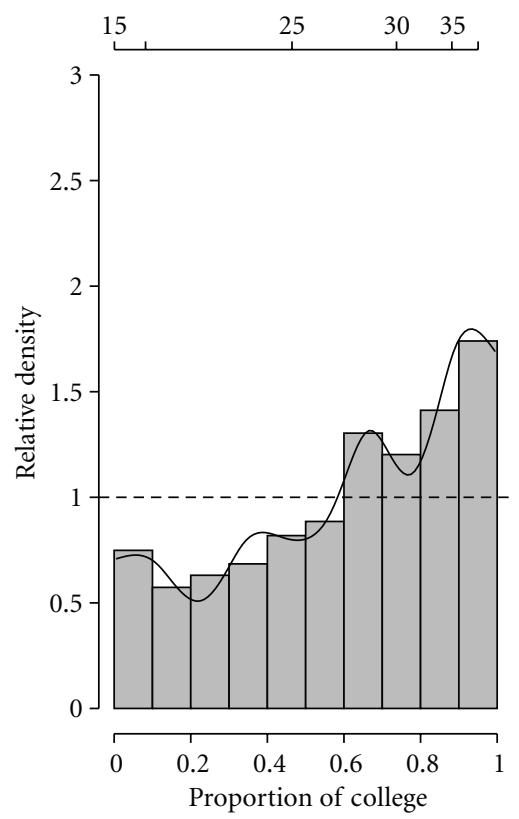

(f) Some college-college entropy $=0.0694$

Figure 3: Age-adjusted relative body mass index distributions (1999-2006) between men (top) and women (bottom) by those with a college degree or more compared to $(\mathrm{a}, \mathrm{d})$ those with less than a high school diploma; (b,e) those with a high school diploma or GED; (c,f) those with some college education. Deciles chart superimposed on relative densities. Data from the National Health and Nutrition Examination Survey.

highest educated women (Figure 3 bottom). Thus, inequality is concentrated in changes in the upper part of the BMI distribution, with a relative hollowing out (i.e., reduced probability) of the distribution in the lowest BMI values (Table 3). Comparing entropy statistics and relative densities shows that the effect is particularly pronounced in those with the lowest education in panel (d), suggesting that education has absolute and relative affects at all levels of education. In other words, the effect of education is strongest in those with the lowest education, but the effect is observed at each education level. From Figure 3, the relative distribution is polarized by the least educated compared to the most 


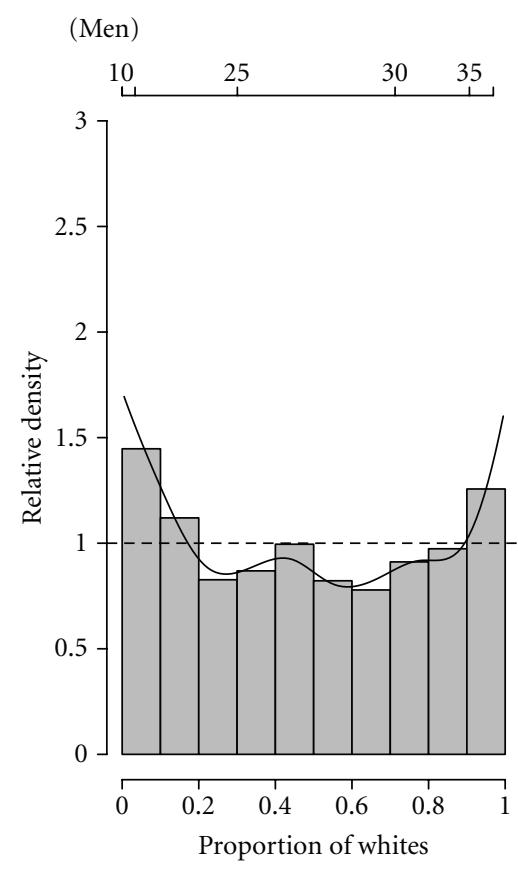

(a) Unadjusted entropy $=0.0228$

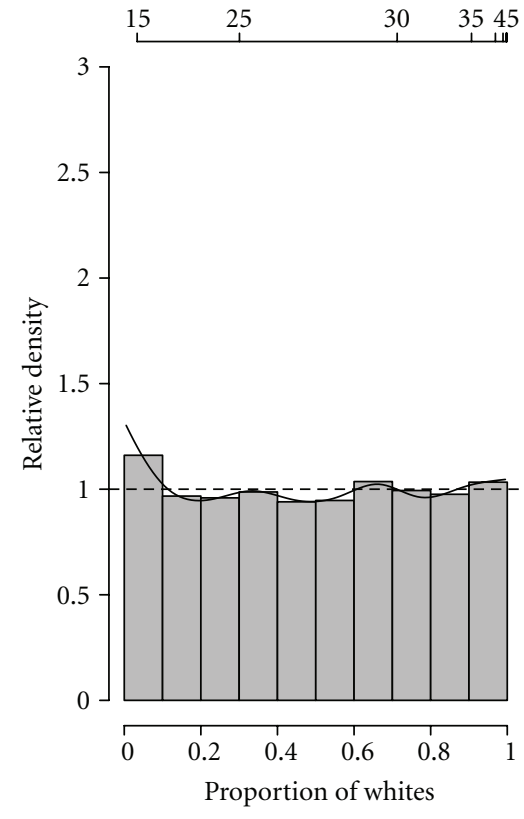

(b) Education effect entropy $=0.00221$

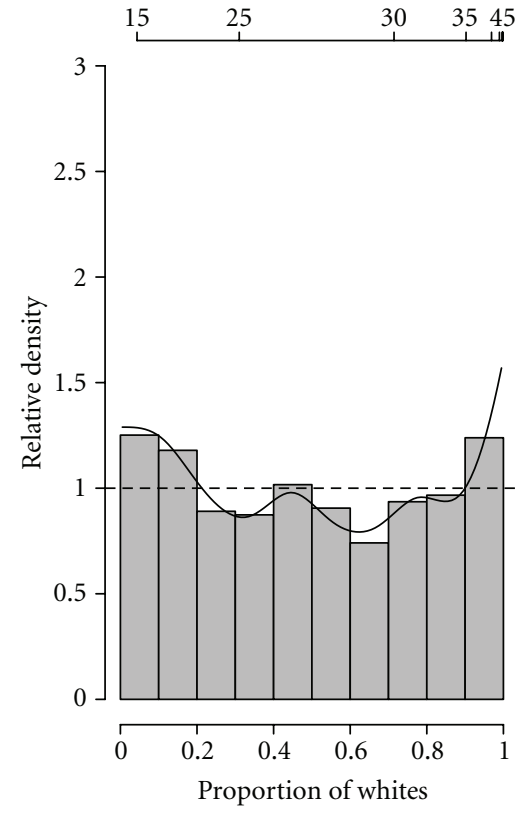

(c) Adjusted entropy $=0.0137$

(Women)

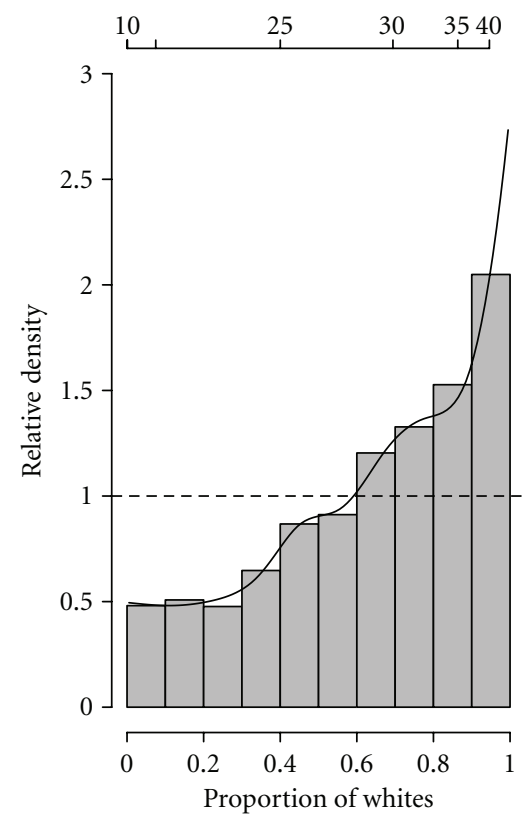

(d) Unadjusted entropy $=0.122$

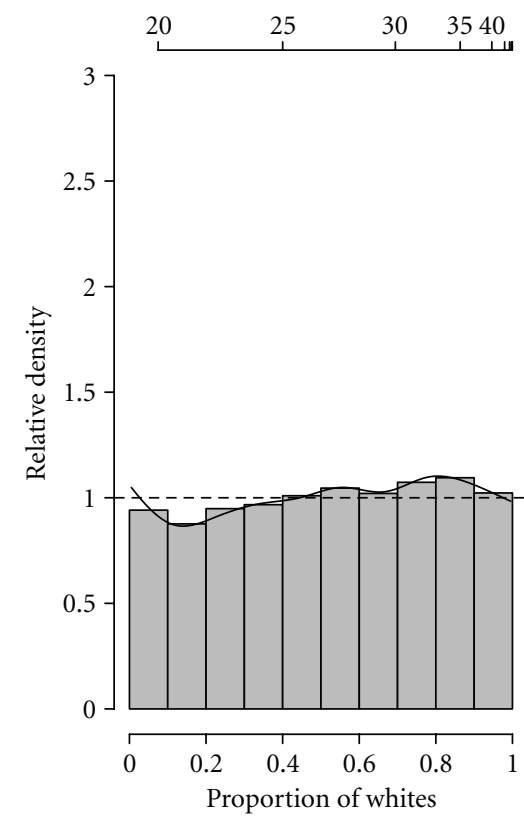

(e) Education effect entropy $=0.00201$

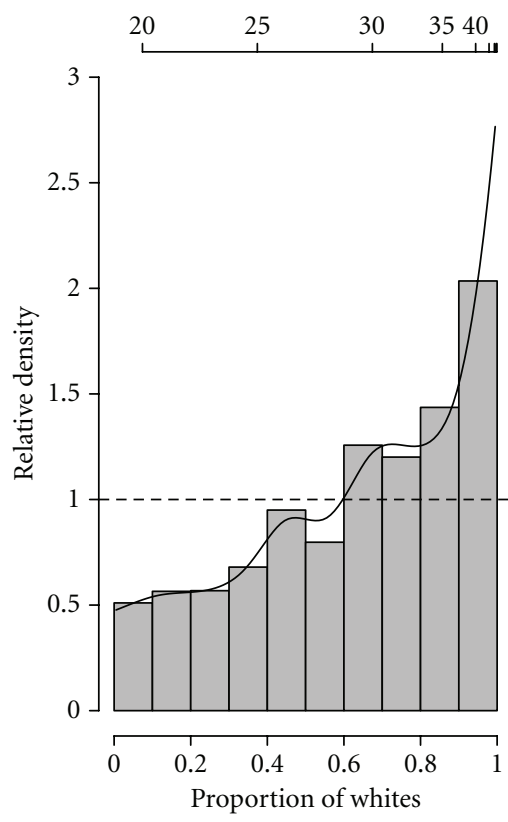

(f) Adjusted entropy $=0.106$

FIGURE 4: Relative body mass index distributions (1999-2006) between non-Hispanic black and white men (top) and women (bottom) adjusted for changes in the education composition between non-Hispanic Blacks and non-Hispanic Whites: (a,d) Unadjusted relative body mass index density; (b,e) effect of changes in the education composition between non-Hispanic blacks and whites; (c,f) education-adjusted relative body mass index density. Deciles chart superimposed on relative densities. Data from the National Health and Nutrition Examination Survey.

educated for men; for women there is a greater proportion of high BMI cases in the less educated groups compared to the most educated.

Figure 4 presents the gender-specific relative distributions comparing non-Hispanic Blacks to Whites compositionally adjusted to have the same distribution of education.
Panel (a, d) represents the unadjusted relative BMI density (same as in Figure $2(\mathrm{a}, \mathrm{d})$ ). The composition effect in panel $(b, e)$ represents the BMI distribution that would have occurred if only the education profile of non-Hispanic Whites had changed (i.e., it represents the effect of changes in the marginal distribution of education). Panel $(c, f)$ 
TABLE 2: Summary measures for distributional comparisons for men, by race, education, and race-education subgroups, United States (1999-2006).

\begin{tabular}{|c|c|c|c|c|c|c|c|}
\hline & \multirow[b]{2}{*}{ Entropy } & \multicolumn{6}{|c|}{ Polarization Index } \\
\hline & & Median & $95 \% \mathrm{CI}$ & Lower & $95 \% \mathrm{CI}$ & Upper & $95 \% \mathrm{CI}$ \\
\hline By Race & .023 & .087 & $.055, .120$ & .067 & $.003, .132$ & .107 & $.043, .171$ \\
\hline \multicolumn{8}{|l|}{ By Edu } \\
\hline Less high school & .046 & .150 & $.108, .193$ & .199 & $.115, .282$ & .102 & $.017, .187$ \\
\hline High School & .035 & .110 & $.069, .151$ & .153 & $.072, .234$ & .068 & $-.014, .149$ \\
\hline Some College & .029 & .087 & $.047, .127$ & .171 & $.091, .250$ & .003 & $-.078, .084$ \\
\hline \multicolumn{8}{|l|}{ By race-edu } \\
\hline Less high school & .036 & .059 & $-.002, .120$ & .042 & $-.079, .162$ & .077 & $-.047, .120$ \\
\hline High School & .022 & .066 & $.001, .131$ & .095 & $-.034, .223$ & .038 & $-.092, .168$ \\
\hline Some College & .019 & .066 & $.005, .128$ & .028 & $-.096, .152$ & .105 & $-.018, .227$ \\
\hline College or greater & .035 & .079 & $-.002, .160$ & .091 & $-.072, .253$ & .066 & $-.095, .228$ \\
\hline \multicolumn{8}{|l|}{ Within race by edu } \\
\hline \multicolumn{8}{|l|}{ NH Whites } \\
\hline Less high school & .039 & .137 & $.085, .189$ & .177 & $.073, .280$ & .098 & $-.007, .202$ \\
\hline High School & .038 & .110 & $.064, .155$ & .150 & $.059, .241$ & .069 & $-.023, .161$ \\
\hline Some College & .032 & .086 & $.040, .132$ & .173 & $.083, .262$ & .000 & $-.092, .092$ \\
\hline \multicolumn{8}{|l|}{ NH Blacks } \\
\hline Less high school & .176 & .108 & $.023, .194$ & .037 & $-.138, .212$ & .179 & $.015, .343$ \\
\hline High School & .096 & .067 & $-.024, .158$ & .056 & $-.128, .241$ & .077 & $-.102, .256$ \\
\hline Some College & .102 & .041 & $-.048, .129$ & -.011 & $-.192, .169$ & .093 & $-.081, .266$ \\
\hline
\end{tabular}

Abbreviations: Edu = education; $\mathrm{NH}=$ non-Hispanic; $\mathrm{CI}=$ confidence interval.

Data from the National Health and Nutrition Examination Survey.

TABLE 3: Summary measures for distributional comparisons for women, by race, education, and race-education subgroups, United States (1999-2006).

\begin{tabular}{|c|c|c|c|c|c|c|c|}
\hline & \multirow[b]{2}{*}{ Entropy } & \multicolumn{6}{|c|}{ Polarization Index } \\
\hline & & Median & $95 \% \mathrm{CI}$ & Lower & $95 \% \mathrm{CI}$ & Upper & $95 \% \mathrm{CI}$ \\
\hline By Race & .122 & .130 & $.099, .161$ & .207 & $.144, .270$ & .053 & $-.008, .115$ \\
\hline \multicolumn{8}{|l|}{ By Edu } \\
\hline Less high school & .123 & .167 & $.125, .210$ & .273 & $.191, .354$ & .062 & $-.023, .146$ \\
\hline High School & .066 & .181 & $.141, .220$ & .309 & $.235, .383$ & .052 & $-.027, .132$ \\
\hline Some College & .069 & .163 & $.125, .201$ & .236 & $.163, .309$ & .089 & $.012, .166$ \\
\hline \multicolumn{8}{|l|}{ By race-edu } \\
\hline Less high school & .113 & .115 & $.053, .176$ & .076 & $-.047, .120$ & .152 & $.031, .274$ \\
\hline High School & .104 & .068 & $.004, .132$ & .102 & $-.028, .232$ & .033 & $-.093, .160$ \\
\hline Some College & .111 & .155 & $.099, .211$ & .246 & $.135, .357$ & .064 & $-.045, .174$ \\
\hline College or greater & .158 & .174 & $.095, .252$ & .282 & $.124, .440$ & .067 & $-.084, .218$ \\
\hline \multicolumn{8}{|l|}{ Within race by edu } \\
\hline \multicolumn{8}{|l|}{ NH White } \\
\hline Less high school & .109 & .152 & $.099, .205$ & .260 & $.156, .364$ & .044 & $-.061, .148$ \\
\hline High School & .067 & .189 & $.145, .233$ & .306 & $.222, .390$ & .073 & $-.017, .162$ \\
\hline Some College & .070 & .158 & $.115, .202$ & .227 & $.142, .312$ & .089 & $.002, .177$ \\
\hline \multicolumn{8}{|l|}{ NH Black } \\
\hline Less high school & .049 & .097 & $.017, .177$ & .158 & $.009, .307$ & .037 & $-.132, .206$ \\
\hline High School & .059 & .100 & $.011, .188$ & .230 & $.065, .394$ & -.030 & $-.215, .155$ \\
\hline Some College & .087 & .183 & $.102, .265$ & .229 & $.071, .386$ & .138 & $-.029, .305$ \\
\hline
\end{tabular}

Abbreviations: $\mathrm{Edu}=$ education; $\mathrm{NH}$ = non-Hispanic; $\mathrm{CI}=$ confidence interval.

Data from the National Health and Nutrition Examination Survey. 


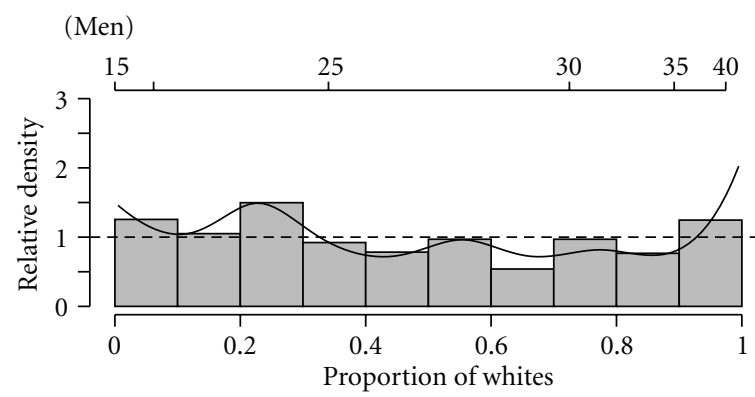

(a) Less HS by race $=0.0359$

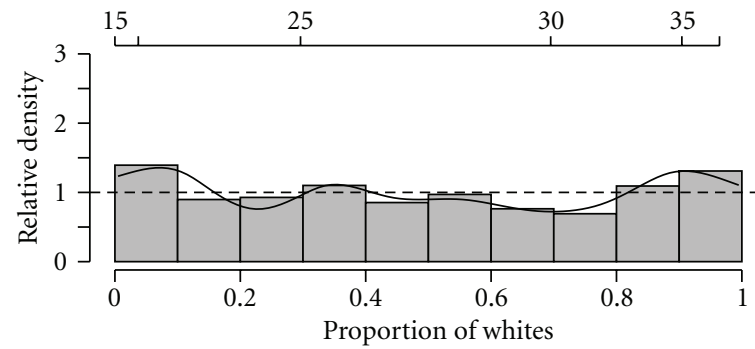

(c) Some college by race entropy $=0.0193$

(Women)

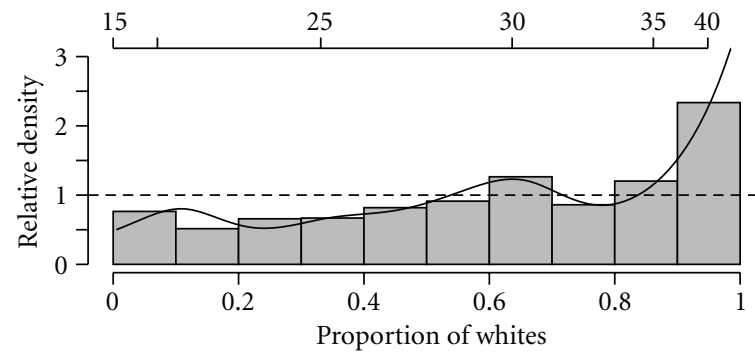

(a) Less HS by race $=0.113$

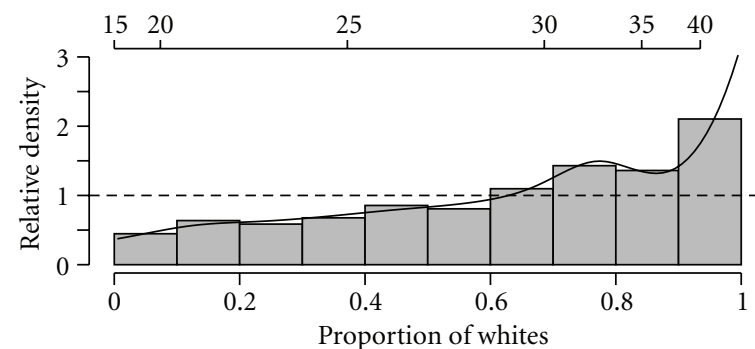

(c) Some college by race entropy $=0.111$

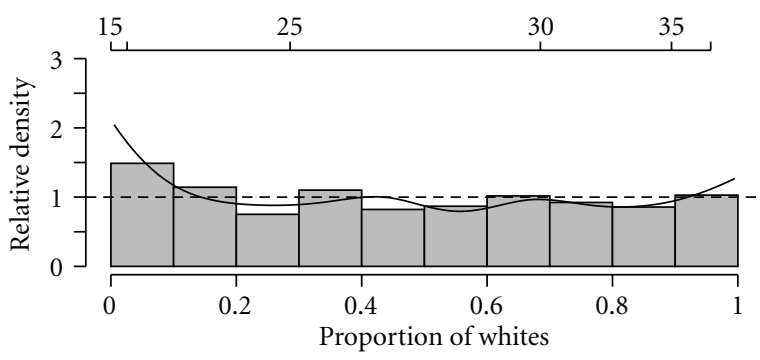

(b) HS by race $=0.219$

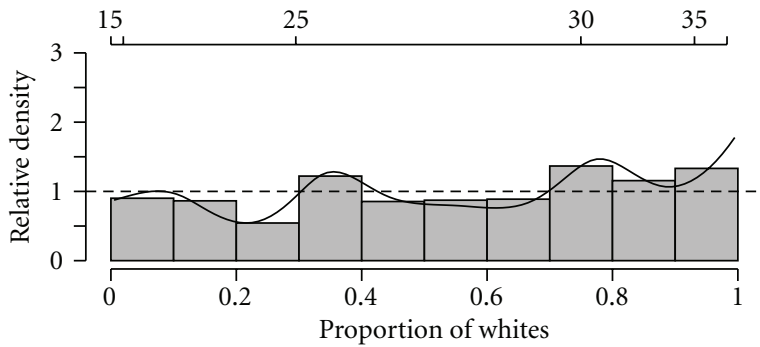

(d) College by race entropy $=0.0346$

(A)

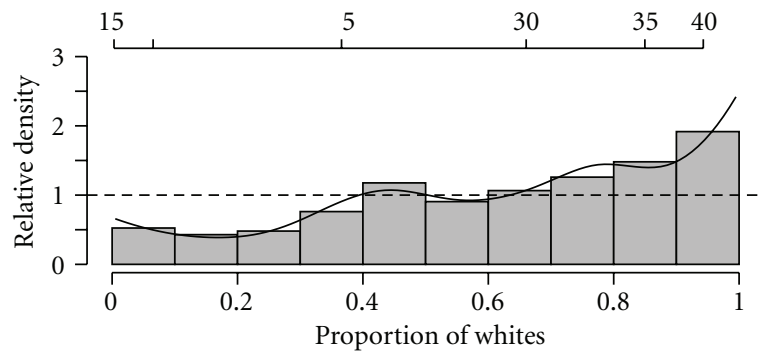

(b) HS by race $=0.104$

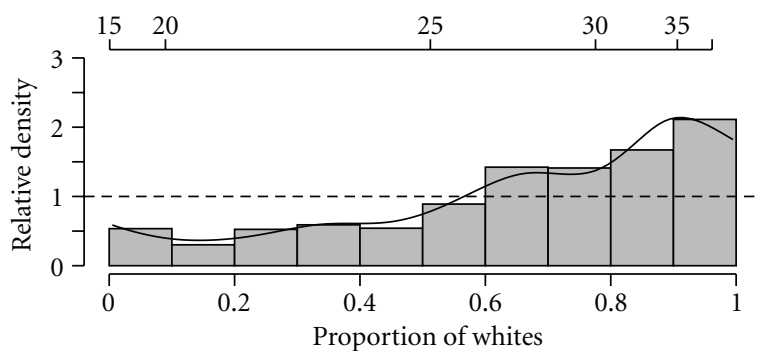

(d) College by race entropy $=0.158$

(B)

FIGURE 5: Relative body mass index distributions (1999-2006) between men (A) and women (B) between non-Hispanic Blacks compared to non-Hispanic Whites by (a) those with less than a high school diploma; (b) those with a high school diploma or GED; (c) those with some college education; (d) those with a college degree or more. Deciles chart superimposed on relative densities. Data from the National Health and Nutrition Examination Survey.

represents the education-adjusted relative density; the expected relative BMI density had the education profiles of non-Hispanic Blacks and Whites been identical (i.e., it represents the residual effect). For men, Figure 4(b) (top) is very close to a uniform distribution, suggesting that differences in the education composition between the two groups have little effect on the relative body mass index distribution. Figure 4(c) (top) is not much different than the original distribution in panel (a) due to the lack of major compositional effects. We can consider the relative sizes of the entropy terms to indicate the relative contributions to the overall difference [15]: the entropy of the residual relative 
density (Figure 4(c) (top)) is .0137, roughly 7 times the size of the composition effect. Further decompositions of the residual effect by location and shape show that it has a location shift slightly to the left (i.e., a steadily declining RD), representing a change in the returns to education (results not shown). The residual is also affected by shape and is more polarized, suggesting that once changes in the median BMI are netted out, non-Hispanic Black men's BMI are more polarized than Whites (results not shown).

For women, Figure 4(e) (bottom) is slightly increasing, suggesting that the differences in the education composition between the two groups lead to a slight shift to the right for non-Hispanic Blacks relative to Whites. The educationadjusted relative density in Figure 4(f) (bottom) still shows a greater proportion of non-Hispanic Blacks in the high BMI range relative to Whites. Further decompositions of the residual effect show a strong location shift to the rightrepresenting a change in the education-BMI relationship for non-Hispanic Blacks relative to Whites (results not shown). The residual is also affected by shape, showing a polarized, U-shaped relative distribution that indicates that, net of median BMI change, non-Hispanic Black women's BMI values are more polarized than White women (results not shown). From Figure 4, the distribution of education does not appear to matter for the racial differences in the BMI distribution for men or women.

A different way to compare the education effects between non-Hispanic Blacks and Whites is to directly compare them within each education category. While the relative density adjusted for education in Figure 4 revealed the educationcomposition effect, Figure 5 presents the gender-specific relative distributions comparing non-Hispanic Blacks to Whites within each education category. These comparisons allow us to look at differences holding education constant across race/ethnic groups - similar to performing a stratified analysis. Differences across education levels then suggest either different returns to education or other factors unrelated to education that are driving the differences between race/ethnic groups. Adjusting for age did not substantively alter the results and are not presented. For men, the comparison varies and is inconsistent across education categories. Non-Hispanic Blacks with a high school degree or some college have a relatively more polarized distribution compared to their similar educated non-Hispanic White counterparts, though this does not hold for those with less than a high school degree or those with a college degree or higher (Table 2).

For women, within each education category there are distributional inequalities among non-Hispanic Blacks compared to Whites (Figure 5). For non-Hispanic Black women with less than a high school degree, there are relatively more cases in the upper tail of the BMI distribution relative to non-Hispanic Whites (Table 3). For non-Hispanic Black women with some college or a college education or more, polarization is driven by changes in the lower tail of the distribution. There are relatively fewer non-Hispanic Black cases in the lowest part of the BMI distribution relative to non-Hispanic Whites (Table 3). From Figure 5, there is little systematic variation in the BMI distribution between race/ethnic-education groups for men; for women with the least education there are relatively more cases in the high BMI range for non-Hispanic Blacks compared to Whites.

Figure 6 presents the gender-specific relative distributions comparing the most educated to each other education group within each race/ethnic group, adjusted for age. Due to limited sample sizes and distributional instability, further decompositions were not conducted. For non-Hispanic White men panel (A), there is greater inequality (particularly in the lower tail) for those with less education compared to the college educated (Table 2). For non-Hispanic Black men (panel (B)), there is a polarized distribution for those with less than a high school diploma or GED relative to the college educated (Table 2).

For non-Hispanic White women (panel (C)), there are distributional inequalities between every education group and the college educated; these differences are driven largely by a lower proportion of low BMI cases in those with less education compared to the college educated (Table 3 ). For non-Hispanic Black women (panel (D)), there are also distributional inequalities between every education group and the college educated; this difference is driven largely by a lower proportion of those with lower education in the lowest parts of the BMI distribution compared to those with a college education. From Figure 6, within non-Hispanic Blacks the BMI distribution is polarized between the least educated and most educated groups for men; for women within each race/ethnic group there are relatively fewer low BMI cases compared to the most educated.

\section{Discussion}

This study used relative distribution methods to explore inequalities in obesity prevalence both between and among gender, race/ethnic, and education subgroups. Relative BMI distributions are fundamentally different by gender. Among men, the relative BMI distribution is polarized by race/ethnicity and those with the lowest education: the least educated groups are disproportionately in the lowest and highest BMI values compared to the most educated; nonHispanic Blacks are also disproportionately in the lowest and highest BMI values compared to non-Hispanic Whites. There is little systematic variation in the BMI distribution between race/ethnic-education groups for men, meaning that there is little difference in the returns of education between race/ethnic groups. However, within non-Hispanic Blacks the relative BMI distribution is also polarized between the least educated relative to the most educated groups.

Among women, the BMI distribution is shifted to the right for non-Hispanic Blacks compared to non-Hispanic Whites. There are also a greater proportion of high BMI cases in the less educated groups compared to the most educated. There are differences in the BMI distribution between race/ethnic groups with the least education, with relatively more cases in the high BMI range for non-Hispanic Black compared to White women. Within race/ethnic groups, for non-Hispanic White and Black women those in the lower education groups have a relative hollowing out 


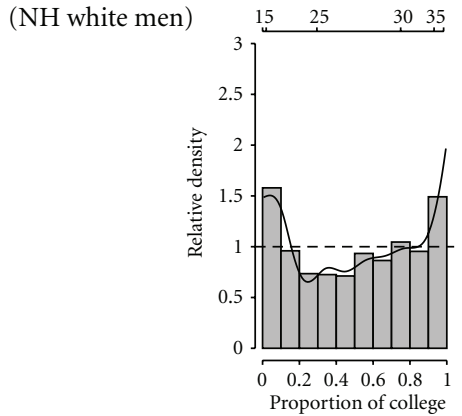

(a) Less HS-college entropy $=0.0385$

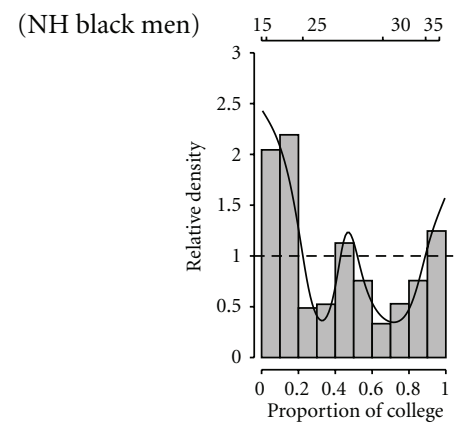

(a) Less HS-college entropy $=0.176$

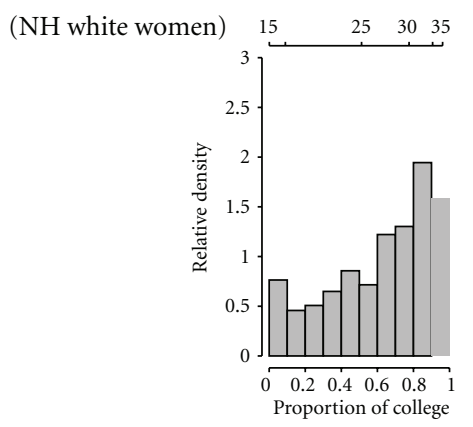

(a) Less HS-college entropy $=0.109$

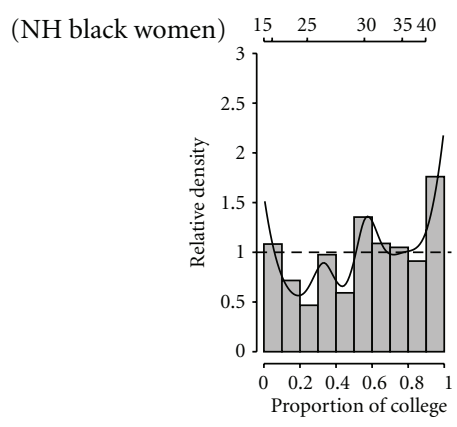

(a) Less HS-college entropy $=0.049$

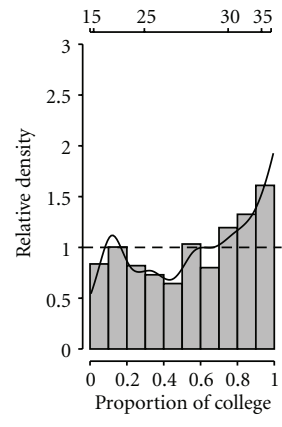

(b) HS-college entropy $=0.0376$

(A)

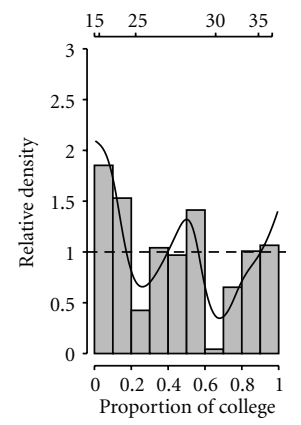

(b) HS-college entropy $=0.0956$

(B)

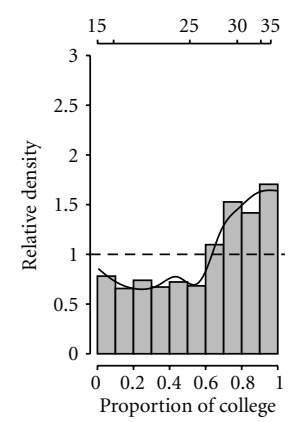

(b) HS-college entropy $=0.0669$

(C)

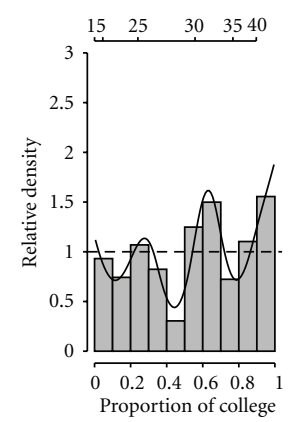

(b) HS-college entropy $=0.059$

(D)

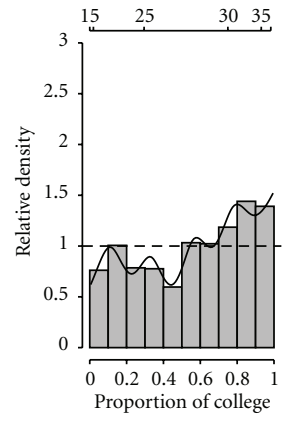

(c) Some college-college entropy $=0.0323$

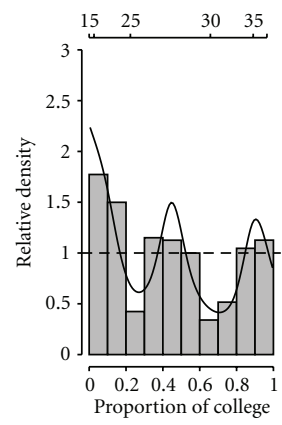

(c) Some college-college entropy $=0.102$

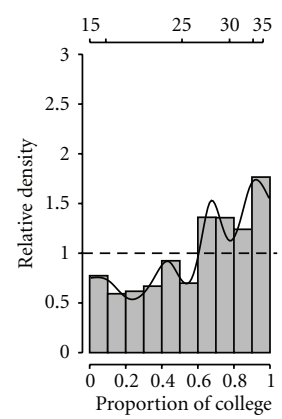

(c) Some college-college entropy $=0.0701$

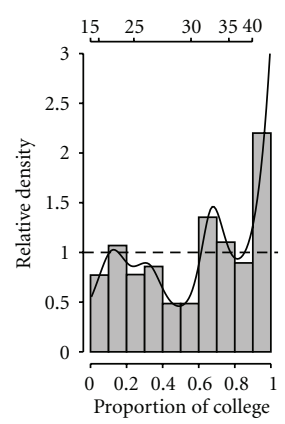

(c) Some college-college entropy $=0.0874$

Figure 6: Age-adjusted relative body mass index distributions (1999-2006) between men (A, B) and women (C, D) and non-Hispanic Whites (A, C) and non-Hispanic Blacks (B, D) by those with a college degree or more compared to (a) those with less than a high school diploma; (b) those with a high school diploma or GED; (c) those with some college education. Deciles chart superimposed on relative densities. Abbreviation: NH: non-Hispanic. Data from the National Health and Nutrition Examination Survey. 
(i.e., reduced probability) of low BMI cases compared to the most educated.

Distributional differences in BMI are summarized by shape differences among men and location differences among women. While this study used cross-sectional data and cannot adjudicate between causal hypotheses, the comparisons presented here can inform hypothesis generation and testing. For instance, for women, comparisons are congruent with a social gradient in BMI [16]. This effect holds over relative and absolute comparisons between race/ethnic groups, education, and race/ethnic-education comparisons. The effect is less pronounced comparing education within race/ethnicity groups. This suggests that the returns affecting the BMI distribution vary between race/ethnic-education subgroups [17].

For men, comparisons were largely differences in shape between race/ethnicity, education, and between education within race/ethnicity groups. Education returns do not vary systematically between race/ethnic groups, and do not explain the polarized relative distribution between nonHispanic Blacks relative to Whites. The remaining polarized relative distributions by race/ethnicity indicate a relative hollowing out of those at disadvantage from the lower and upper thirds of the BMI distribution, and a resulting increase in the extreme tails of the BMI distribution of both underweight and obesity. These comparisons suggest that mechanisms are affecting different parts of the BMI distribution differently. Further, they suggest that the mean of the BMI distribution is less representative for men, and that greater attention needs to be paid to inequality within groups rather than across them [18]. For instance, polarized relative distributions are congruent with neighborhood and economic insecurity mechanisms affecting diet quality, where those at disadvantage are at increased risk of both underweight and obesity [6]. Polarized relative distributions are also important in understanding the risks to these vulnerable populations. Studies have found increased mortality for both people with high and low normal BMI values [19-21].

This study has several strengths. First, by using a nationally representative population and including both men and women, this study allows for a detailed assessment of BMI inequality. Second, using exploratory approaches and distribution-oriented analyses allows assessment of inequalities in the burden of obesity within and among groups, the timing of BMI distributional changes, and whether these trends are statistically significant. Similar work by Zhang and Wang using the concentration index on socioeconomic status found that inequality varied by gender, age, and ethnicity [9]. A strength of their study and this one is that they consider income or education as relative rankings instead of absolute values. This helps to partially eliminate the confounding of ethnicity (i.e., differential returns) on the relationship between SES/education and obesity. Third, a comparison using the full BMI distribution avoids categorizing risk into discrete categories (e.g., overweight or obese).

Several properties of the relative distribution make it favorable to study BMI distributional inequality. First, we might expect that along the distribution BMI values may go up or down over time; relative distribution methods can handle these negative values (which neither the GINI nor Lorenz curve can accommodate) [15]. Second, since relative social status is a predictor of obesity, relative distributions make these comparisons explicit. Third, absolute differences in BMI also matter (even a modest reduction in BMI is associated with improved morbidity $[3,21]$ ) and are also captured by the relative distribution. Fourth, the robust handling of outliers in relative distribution methods is important since individuals at the tails (e.g., those with extreme obesity) suffer greater morbidities [3,21]. Relative distributions are robust to outliers because of the rescaling of the comparison to the referent distribution and the lack of parametric assumptions [12]. Fifth, comparisons can be summarized graphically as well as using summary statistics can be decomposed by location and shape and can control for covariates.

There are also several limitations in using relative distributions. First, comparisons can quickly become unwieldy in controlling for many covariates. For instance, this study omitted Mexican Americans due to the large number of comparisons that would be presented. Second, while not an issue with the measured height and weight data in this study, measurement techniques that affect distributions can limit the utility of relative distributions. For example, selfreported data that are prone to data heaping can severely alter the quantiles of a distribution [12]. Third, particularly when controlling for many covariates or conducting decompositions, a large enough sample is necessary to ensure distributional stability [12]. In this study, the withinrace/ethnicity comparisons by education demonstrated these difficulties by more unstable relative densities.

\section{Conclusions}

This study highlights potential BMI-related processes that differ significantly by gender, race, and education. Moreover, it is likely that prior work on mean differences in BMI do not tell the whole story; these processes have affected the parts of the BMI distribution within as well as between groups differently. Further research is needed to explore the fundamental differences in the BMI distribution by gender, and to account for the remaining race/ethnic differences net of education (particularly for men). By identifying the differences in distributions, epidemiologists can focus empirical work on accounting for the mechanisms generating BMI inequalities within different parts of the distribution.

\section{Conflict of Intrests}

The author has no conflict of interests to disclose.

\section{References}

[1] K. M. Flegal, M. D. Carroll, C. L. Ogden, and L. R. Curtin, "Prevalence and trends in obesity among US adults, 19992008," Journal of the American Medical Association, vol. 303, no. 3, pp. 235-241, 2010. 
[2] C. L. Ogden, M. D. Carroll, M. A. McDowell, and K. M. Flegal, "Obesity among adults in the United States-no change since 2003-2004," NCHS data brief 1, National Center for Health Statistics, Hyattsville, Md, USA, 2007.

[3] NIH, NHLBI Obesity Education Initiative, "Clinical Guidelines on the Identification, Evaluation, and Treatment of Overweight and Obesity in Adults," 1998, http://www.nhlbi.nih.gov/guidelines/obesity/ob_gdlns.pdf.

[4] C. L. Ogden, M. D. Carroll, L. R. Curtin, M. A. McDowell, C. J. Tabak, and K. M. Flegal, "Prevalence of overweight and obesity in the United States, 1999-2004," Journal of the American Medical Association, vol. 295, no. 13, pp. 1549-1555, 2006.

[5] A. Drewnowski and S. E. Specter, "Poverty and obesity: the role of energy density and energy costs," American Journal of Clinical Nutrition, vol. 79, no. 1, pp. 6-16, 2004.

[6] N. Darmon and A. Drewnowski, "Does social class predict diet quality?" American Journal of Clinical Nutrition, vol. 87, no. 5, pp. 1107-1117, 2008.

[7] G. L. Burke, D. R. Jacobs, J. M. Sprafka, P. J. Savage, S. Sidney, and L. E. Wagenknecht, "Obesity and overweight in young adults: the CARDIA study," Preventive Medicine, vol. 19, no. 4, pp. 476-488, 1990.

[8] J. Sobal and A. J. Stunkard, "Socioeconomic status and obesity: a review of the literature," Psychological Bulletin, vol. 105, no. 2, pp. 260-275, 1989.

[9] Q. Zhang and Y. Wang, "Socioeconomic inequality of obesity in the United States: do gender, age, and ethnicity matter?" Social Science and Medicine, vol. 58, no. 6, pp. 1171-1180, 2004.

[10] Y. Wang and M. A. Beydoun, "The obesity epidemic in the United States-gender, age, socioeconomic, racial/ethnic, and geographic characteristics: a systematic review and metaregression analysis," Epidemiologic Reviews, vol. 29, no. 1, pp. 6-28, 2007.

[11] P. Contoyannis and J. Wildman, "Using relative distributions to investigate the body mass index in England and Canada," Health Economics, vol. 16, no. 9, pp. 929-944, 2007.

[12] M. S. Handcock and M. Morris, "Relative distribution methods," Sociological Methodology, vol. 28, no. 1, pp. 53-97, 1998.

[13] Centers for Disease Control and Prevention (CDC) and National Center for Health Statistics (NCHS), National Health and Nutrition Examination Survey Data, U.S. Department of Health and Human Services, Centers for Disease Control and Prevention, Hyattsville, Md, USA, 1999-2006.

[14] R Development Core Team, R: A language and environment for statistical computing, R Foundation for Statistical Computing, Vienna, Austria, 2008.

[15] M. S. Handock and M. Morris, Relative Distribution Methods in the Social Sciences, Springer, New York, NY, USA, 1999.

[16] M. Marmot, "Inequalities in health," New England Journal of Medicine, vol. 345, no. 2, pp. 134-136, 2001.

[17] M. D. Hayward, T. P. Miles, E. M. Crimmins, and Y. Yang, "The significance of socioeconomic status in explaining the racial gap in chronic health conditions," American Sociological Review, vol. 65, no. 6, pp. 910-930, 2000.

[18] K. T. Leicht, "Broken down by race and gender? Sociological explanations of new sources of earnings inequality," Annual Review of Sociology, vol. 34, pp. 237-255, 2008.

[19] A. Romero-Corral, V. M. Montori, V. K. Somers et al., "Association of bodyweight with total mortality and with cardiovascular events in coronary artery disease: a systematic review of cohort studies," Lancet, vol. 368, no. 9536, pp. 666$678,2006$.
[20] A. Oreopoulos, R. Padwal, K. Kalantar-Zadeh, G. C. Fonarow, C. M. Norris, and F. A. McAlister, "Body mass index and mortality in heart failure: a meta-analysis," American Heart Journal, vol. 156, no. 1, pp. 13-22, 2008.

[21] Prospective Studies Collaboration, "Body-mass index and cause-specific mortality in 900000 adults: collaborative analyses of 57 prospective studies," The Lancet, vol. 373, no. 9669, pp. 1083-1096, 2009. 


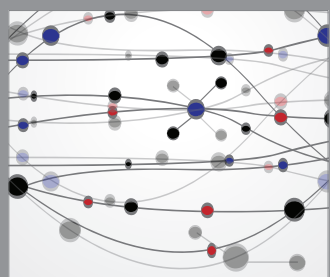

The Scientific World Journal
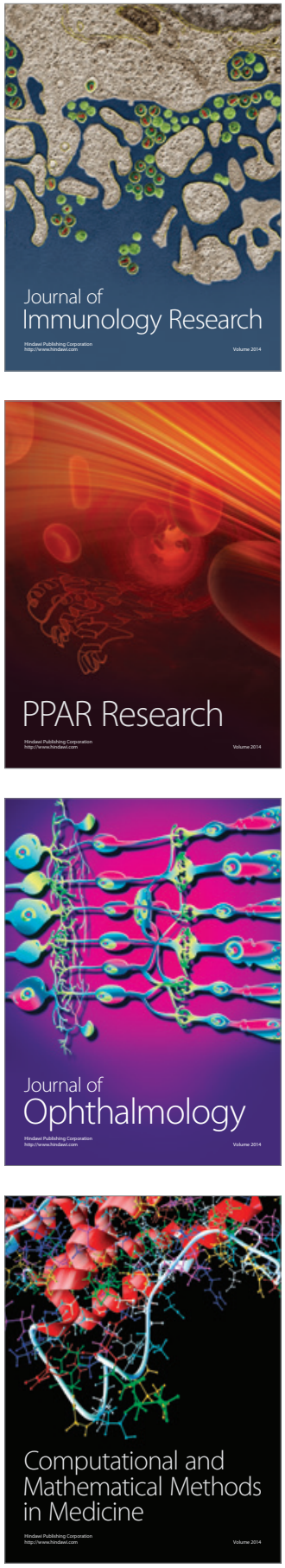

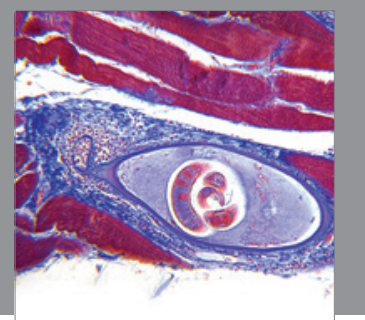

Gastroenterology

Research and Practice
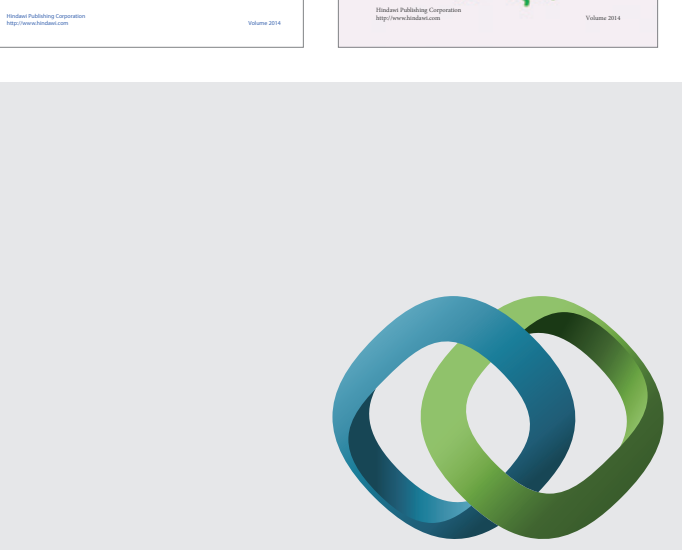

\section{Hindawi}

Submit your manuscripts at

http://www.hindawi.com
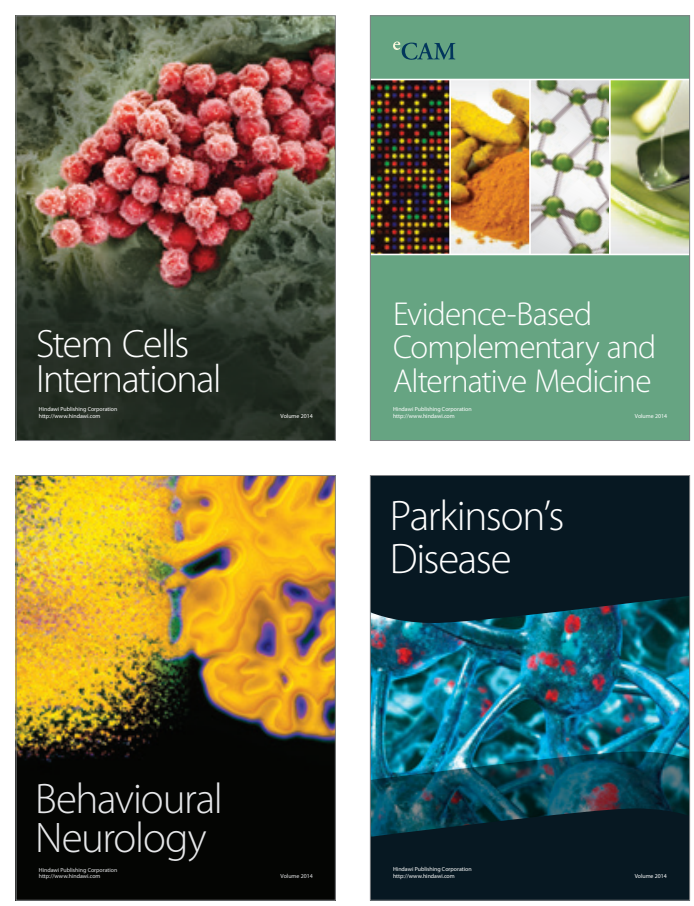

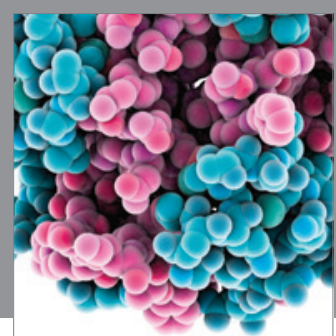

Journal of
Diabetes Research

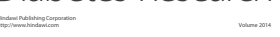

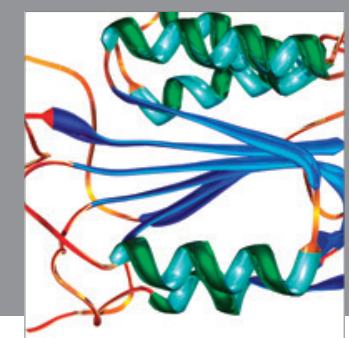

Disease Markers
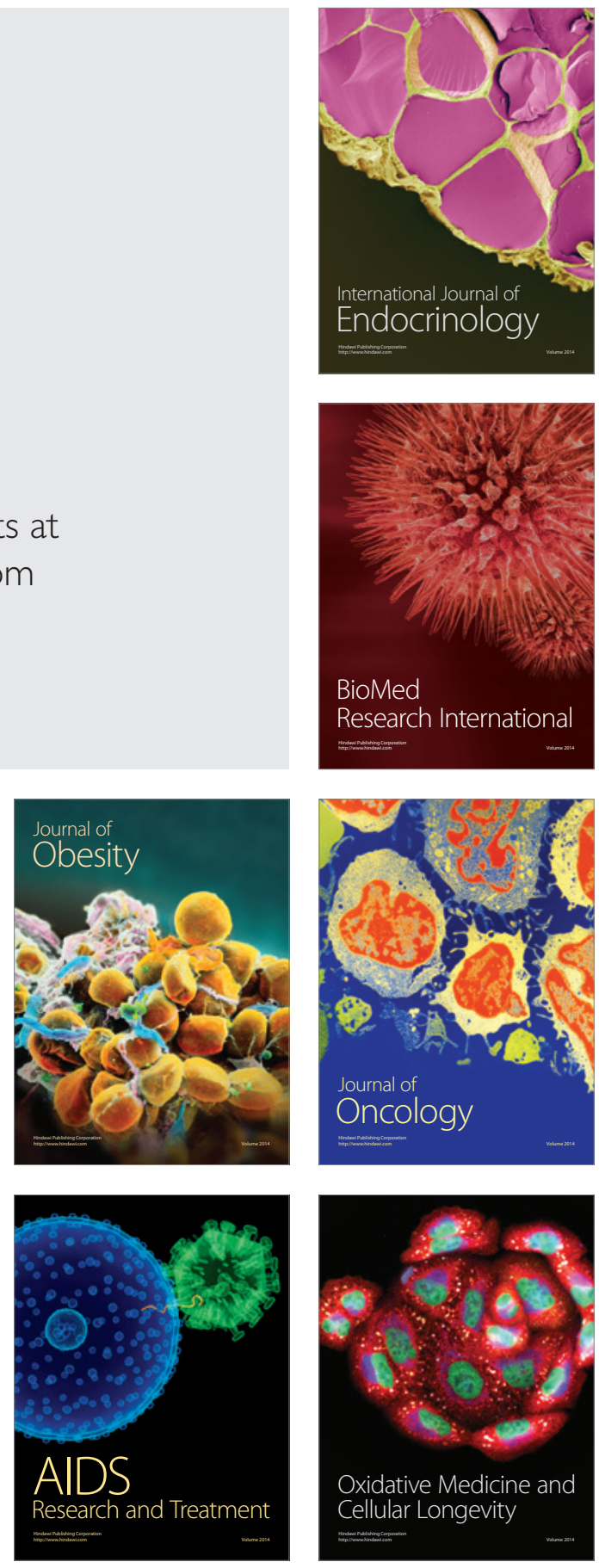